\title{
Recombinant TMV Vector for Production of Highly Immunogenic Plant-Based Vaccine Antigens Against Herpes Simplex Virus Type 2
}

Ahmed K. El-Attar ${ }^{a, d}$, Aboul-Ata E. Aboul-Ata ${ }^{a}$, Josefine Persson ${ }^{b}$, Hamed H. Mazyad ${ }^{a}$, Ali M. Harandi ${ }^{b}$, and Olof Olsson ${ }^{\mathrm{c}, \mathrm{d}}$

aDepartment of Plant Virus and Phytoplasma Research Section, Plant Pathology Research Institute, ARC, P.O. Box 12619, Giza Egypt.

${ }^{b}$ Department of Microbiology and Immunology, Institute of Biomedicine, University of Gothenburg, Medicinaregatan 7A, SE40530, Gothenburg, Sweden.

${ }^{c}$ Department of Pure and Applied Biochemistry, Lund University, Getingevägen 60, Box 124, SE22100 Lund, Sweden.

'Department of Plant and Environmental Sciences, Carl Skottsbergsgata 22B, Box461, University of Gothenburg, SE40530 Gothenburg, Sweden.

\section{A B S T R A C T}

Genital herpes disease is caused mainly by herpes simplex virus type 2 (HSV-2), which is sexually transmitted with a high prevalence in both developed and developing countries. No vaccine is currently available against genital herpes. In this study, we introduced and expressed two genes encoding HSV-2 glycoprotein D (gD) and VP16 protein and a GFP marker gene (control) in tobacco seedlings. Positive plant infection was assessed through characteristic tobacco mosaic virus disease symptoms on leaves and by monitoring the fluorescence emitted by the expressed GFP protein. Expression of the HSV-gD2 and VP16 antigens was verified by RT-PCR, ELISA and Western blot. As a proof of concept, the immunogenicity and the protection ability of the plant produced gD antigen was tested in a mouse model of genital herpes and compared to $\mathrm{gD}$ antigen produced in a mammalian expression system. This showed that the plantgD preparation, when used in combination with a $\mathrm{CpG}$ oligodeoxynucleotide as adjuvant, was highly immunogenic and capable of inducing complete immunity to lethal vaginal HSV-2 challenge in mice. Thus, the data presented here may have implications for the development of a production system for highly immunogenic plant-based HSV-2 vaccine antigens.

Keywords: TMV, plant vaccine, génital herpes, HSV-2, gD, VP16, GFP, mouse model genital herpes

\section{Introduction}

Herpes Simplex Virus has 8 types; i.e. 1 and 2 (HSV-1, HSV-2) are infect human and closely related. They are belong to the Alphaherpesvirinae subfamily (Davison et al., 2009). HSV-1 is commonly associated with oral lesions, although genital lesions are also frequent found. HSV-2 is, the most common, causal of genital ulcers, with an estimated 536 million prevalent and 24 million global incident infections in the age group 15-49 years. Moreover its incidence is increasing in both developing and developed countries (Abd-Elshafy and Bahgat; 2017). Both HSV-1 and HSV-2 can induce additional severe symptoms such as meningitis and neonatal infections (Basse et al., 
2008). HSV-2 infection is considered as risk factor for HIV-1 transmission, especially developing countries (Glynna, 2009). Statistics from World Health Organization (WHO) show that HSV-2 prevalence in HIV-infected women is increasing in US and Europe as well as in North Africa, including Egypt (Bahaa et al., 2017). Furthermore, HSV-2 infection is increasing with age and is higher in females than in males (Looker et al., 2008).

Big efforts have been made to develop genital herpes vaccines throughout last decades. Traditional vaccine development, such as attenuated viruses, have shown to confer protective immunity in different animal models of genital herpes. The viral envelope consists of several proteins those are highly immunogenic (Awasthi et al., 2013). Several recombinant HSV envelope glycoproteins have been tested in combination with an adjuvant for immunogenicity (Jazayeri et al., 2009). In particular, the $\mathrm{gB}$ and $\mathrm{gD}$ envelope glycoproteins have been targeted and tested in animal models (Bernstein and Stanberry, 1999; Awasthi et al., 2014). Other target genes for vaccines have been studied in HSV-2 tegument proteins like e.g. VP16, which stimulates transcription of the HSV immediate-early genes that are essential for HSV-2 replication (Dutton et al., 2013; Aboul-Ata et al., 2014). Such HSV protein subunit vaccines, prophylactically or therapeutically administered, have shown to elicit antigen-specific immune responses in humans. However, despite numerous efforts they have displayed a poor protective immunity. In addition, the application of these vaccines in humans also has safety concerns. Consequently no vaccine to counter genital herpes in humans is currently available on the market (Dutton et al., 2013). Thus there is a great need to further develop and extend present strategies to prevent genital herpes infection (EITurkey et al., 2014).

During recent years plant-based alternative systems to produce anti-viral vaccines have been developed (El Attar et al., 2004; Claire et al., 2011). Plant production systems have the advantage of well-established methods to harvest and process plant derived products, guaranteed absence of human pathogens in the final product and superior ways to stabilize the product for storage purposes (Yusibov, 2013; Regnard et al., 2017). The production can easily be scaled up to at an industrial scale and this to a lower cost than any animal production systems (Lal et al., 2007; Jain et al., 2013; Laere et al., 2016). Other advantages with plant cells are their capability of various post-translational protein modifications like glycosylation, in contrast to bacterial production systems (Takeyama, et al. 2015; Guan, 2013). However, since the exact modification could differ 
in plants and animals, both plant- and animal-produced antigens will have to be tested in a relevant animal model system to prove their activity (Yang, et al, 2017).

Given this prerequisites, vaccine production systems should be especially suitable for developing countries, provided they can be accepted from a socio-culturally point of view (Chan and Daniell, 2015). Here we describe the production of plant derived HSV$2 \mathrm{gD}$ and VP16 antigens using a transient expression system based on a genetically modified tobacco mosaic virus (TMV) (Shivprasad, et al, 1999 and Nemchinov, et al, 2000). As a proof of concept, the immunogenicity and the protection ability of the plant produced $\mathrm{gD}$ antigen was tested in a mouse model of genital herpes in parallel with a HSV-2 gD protein produced in a mammalian expression system

\section{Materials and methods}

\subsection{Plant growth}

Tobacco, Nicotiana benthamiana,seeds were kindly provided by Dr. Christer Olsson, University of Gothenburg, Sweden. The plants were grown under metal halogen lamps (HQI-TS $400 \mathrm{~W} / \mathrm{DH}$, Osram, Munich, Germany) giving a photon flux density of 240 $\mu \mathrm{mol} / \mathrm{m}^{-2} / \mathrm{sec}^{-1}$ at an $18 \mathrm{~h}$ photoperiod, a day/night temperature of $24 / 26^{\circ} \mathrm{C}$ and a relative humidity of about $70 \%$. The plants were watered as required.

\subsection{Cloning and vector construction}

HSV-2 DNA was prepared from the HSV-2 strain 333 using the NucleoSpin virus kit (Clontech Laboratories Inc.). The gD and VP16 genes were PCR amplified from the HSV-2 DNA according to standard protocols using the following primers:

gD forward, 5'-CCCTITAATTAATGGGGCGTTTGACCTCC-3'.

VP16 forward, 5'-CGGTTAATTAATGGACCTGTTGGTCGACG-3'.

gD reverse, 5'-CCCTCGAGTTAGATCAGGCCCGGGTTGCT-3'.

VP16 reverse; 5'-GGCTCGAGCTACCCCCCAAAGTCGTCAAT-3'.

Pacl or Xhol restriction enzyme sitess were included in the primers (underlined).

Platinum $\mathrm{Hi}$ fidelity Taq DNA polymerase (Invitrogen) was used for all PCR amplifications. The PCR products were, separated on a $0.7 \%$ agarose gel and fragments of predicted sizes were, isolated and cloned into the plasmid vector $\mathrm{pCR} \otimes 2.1$ TOPO (Invitrogen Cat. No: K4520-01). This generated, in addition to the GFP plasmid, denoted pAKGFP, two other plasmids denoted pAKgD and pAKVP16. All plasmids were transformed into TOP-10 E. coli cells (Ausubel et al; 1999), amplified and purified by the 
QIAprep Spin Miniprep Kit from Qiagen. The obtained recombinant plasmids were analyzed with restriction digestions and verified by DNA sequencing. After this, the gD, VP16 and GFP fragments were excised from the recombinant TOPO vectors and cloned into the Xhol-Pacl sites of the engineered tobacco mosaic virus vector 30B (TMVTMGMV) (Shivprasad, et al, 1999) forming the recombinant TMV viruses AKTMVgD, AKTMVP16 and AKTMVGFP (Fig. 1). Again, the cloning steps were verified by DNA sequencing. The original GFP clone as well as the 30B (TMV-TMGMV) vector were kindly provided by Dr. Shailaja Shivprasad from the Farunhofer Center for Molecular Biotechnology; DE; USA.

\subsection{Plant transfection}

The chimeric AKTMVgD, AKTMVP16 and AKTMVGFP viruses (Fig.1) were linearized by the Sapl restriction enzyme and in vitro transcribed by T7 RNA polymerase using the RNAMaxx High Yield Transcription system from Stratagene. Synthesized transcripts were then capped by the RNA cap-structure analogue $7 G(5) p p p(5) G$, provided by Invitrogen and the capped transcripts were dissolved in 1X PBS (0.1 M phosphate, and $0.15 \mathrm{M}$ sodium chloride, $\mathrm{pH} 7.2$ ). After abrading the leaf surface of three-week old $N$. benthamiana tobacco plants with celite powder, the capped transcripts were gently rubbed into the upper leaves. This created a local infection that manifested itself as a systemic infection after about 2 weeks.

\subsection{Expression analysis of chimeric genes}

Total RNA was extracted from locally and systemically infected leaves showing visual symptoms, using the RNA-easy Plant Mini Kit (Qiagen) according to the manufactures guidelines. The extracted RNA was used as template for one-tube RT-PCR reverse transcription and amplification using the OneStep RT-PCR Kit (QIAGEN). Forward primers specific for either $\mathrm{gD}$ or VP16 were used with a complementary primer specific for the TMV vector (5'-CGCGATCCAAGACACAACCCTT-3') located 90 bases downstream the Xhol restriction site used to insert the HSVII genes.

Protein was extracted from leaf samples homogenized in 1X PBS containing 1\% plant protease inhibitor cocktail (Sigma) and centrifuged $2 \times 14000 \mathrm{rpm}$ for $10 \mathrm{~min}$ at $4 \stackrel{\circ}{\circ} \mathrm{C}$ in an Eppendorf centrifuge. The supernatant fraction was mixed 1:1 with sample buffer and proteins were analyzed by $10-20 \%$ Tris-glycine PAGE (Novex). In Western blot experiments, proteins were electro-transferred at $80 \mathrm{mV}$ to $0.2 \mu \mathrm{m}$ nitrocellulose membranes (Bio Rad USA) using the Sigma Aldrich electro-blotter system. The 
membranes were incubated with an 1:1000 dilution of a mouse monoclonal HSVgD antibody (Abcam), an 1:200 dilution of VP16 (1-21) mouse monoclonal antibody (Santa Cruz) or an 1:500 dilution of anti TMV polyclonal antibody (DSMZ-Germany). ECL Sheep Anti-Mouse IgG Horse Radish Peroxidase-conjugated whole antibody (GE Healthcare) was used at a 1:2000 dilution as secondary antibody. Specific signals from bound antibodies were developed using an Immune-Star HRPC chemiluminescence detection kit obtained from BioRad, USA. In a separate experiment a TMV polyclonal antibody (DSMZ, Germany) was used as the primary antibody and ECL Donkey AntiRabbit antibody (Santa Cruz) as the secondary antibody to confirm the systemic infection of TMV virus like particles (VLP).

As for the ELISA experiments, microtiter plate wells were coated with $200 \mu$ l of a 1:200 dilution of anti-TMV polyclonal antibodies (DSMZ, Germany) dissolved in carbonate buffer $\left(50 \mathrm{mM} \mathrm{Na}_{2} \mathrm{CO}_{3}, \mathrm{pH}\right.$ 9.6). After incubating for $2 \mathrm{~h}$ at $37^{\circ} \mathrm{C}$ the wells were blocked for $1 \mathrm{~h}$ at $37^{\circ} \mathrm{C}$ with $1 \% \mathrm{BSA}$ solution containing $0.1 \%$ Tween 20 . The plates were then incubated overnight at $4^{\circ} \mathrm{C}$ with the plant extracts, followed by incubation with anti-gD mouse monoclonal antibodies (MAbs), anti VP16 mouse MAbs or anti-TMV polyclonal antibodies (DSMZ- Germany) at $37^{\circ} \mathrm{C}$ for $2 \mathrm{~h}$. After washing 5 times with $1 \mathrm{X}$ PBS-Tween buffer between the different incubations, anti-mouse IgG-HRP or anti rabbit IgG-AP conjugates were added. After incubating at room temperature for $2 \mathrm{~h}$, horseradish peroxidase or alkaline phosphatase substrates were added to the wells. Plates were read at $405 \mathrm{~nm}$ using an ELISA reader (ELX 800, Biotek Instruments, Inc.). To detect GFP, a Zeiss Axioscope with a 40X Carl Zeiss Plan-Neofluar objective (N.A. $0.75 \mathrm{~mm}$ ), a Zeiss filter set (green excitation filter 450-490 HB) and a Zeiss AttoArc2 HBO-100W mercury lamp was used. Images were captured with an AxioCam camera and processed with the AxioVision 4.6.3 Imaging System and Adobe Photoshop software. The exposure time was $10 \mathrm{~ms}$.

For mice immunization experiments, extracted protein was purified using ÄKTA pure protein purification system utilizing Sephacryl ${ }^{\mathrm{TM}}$ High Resolution resins HiPrep ${ }^{\mathrm{TM}}$ Sephacryl HR columns (GE Healthcare, Sweden).

\subsection{Mouse immunization experiments}

Seven- to nine-weeks old female C57BL/6 mice were purchased, from Charles River Laboratories (Germany), and kept in ventilated cages under specific pathogen-free conditions, at the EBM Animal Facility, Sahlgrenska Academy at University of 
Gothenburg. All animal experiments were carried out with the approval of the Ethical Committee for Animal Experimentation in Gothenburg, Sweden (311/12).

\subsection{Immunization scheme}

Groups of mice received intranasal immunization three times at seven-day intervals. At each immunization occasion the mice were inoculated, using a fine micropipette tip, with $4 \mu \mathrm{g}$ purified recombinant HSV-2 gD expressed in either plant or Chinese Hamster Ovary cells (Mammalian Protein Expression Facility at Sahlgrenska Academy, University of Gothenburg) together with $15 \mu \mathrm{g}$ of the Toll-like receptor 9 agonist CpGODN1826 (MWG Eurofins). Immunizations were performed after the mice had been anesthetized with isoflurane (Baxter Medical AB, Sweden). Dilutions were made in sterile phosphate-buffered saline (PBS) and a control group was given PBS alone.

\subsection{Collection of blood samples}

Blood samples were drawn from the tail vein two weeks after the final immunization. The samples were stored at $4^{\circ} \mathrm{C}$ overnight, after which the sera were separated by two centrifugations at $2500 \mathrm{rpm}$ for $5 \mathrm{~min}$ (Thermo Scientific, Fresco 17) and frozen at $20^{\circ} \mathrm{C}$.

\subsection{Analysis of specific $\lg G$ antibody response}

An ELISA-based analysis was used to measure gD-specific $\lg G$ in the sera collected from the immunized mice. Low-binding 96-well plates (Nunc) were coated with gD (5 $\mu \mathrm{g} / \mathrm{ml}$ ) diluted in $50 \mathrm{mM}$ carbonate buffer $(\mathrm{pH}$ 9.6). The plates were incubated at room temperature overnight, blocked with PBS-BSA (2\%) for $30 \mathrm{~min}$ at $37^{\circ} \mathrm{C}$ and washed with PBS-Tween20 (0.05\%).

Frozen sera were thawed and diluted 1:10 in PBS-Tween20 (0.05\%) containing 1\% BSA. An1:3 dilution of each sample was added to the washed plates, which then were incubated at $37^{\circ} \mathrm{C}$ for $1 \mathrm{~h}$. After washing with PBS-Tween20 (0.05\%), HRP-conjugated goat a-mouse IgG (Jackson Immuno-Research Laboratories, Inc., USA), IgG1 or IgG2c (Southern Biotech, USA) were added and the plates were incubated at $37^{\circ} \mathrm{C}$ for one more hour. The plates were then washed with PBS-Tween20 (0.05\%) and $1 \mathrm{mg} / \mathrm{ml} \mathrm{o}$ phenylenediaminedihydro-chloride (Sigma Aldrich) in citrate buffer $(0.1 \mathrm{M}, \mathrm{pH} 4.5)$, containing $\mathrm{H}_{2} \mathrm{O}_{2}(0.04 \%)$, was added to develop the signal. After incubation, at room temperature in the dark, the development was stopped with $25 \mu \mathrm{l} \mathrm{H}_{2} \mathrm{SO}_{4}(1 \mathrm{M})$. The 
absorbance was read at $490 \mathrm{~nm}$ (Spectra Max 250) and data analyzed with SoftMax Pro 4 software.

\subsection{HSV-2 vaginal challenge}

Mice were injected subcutaneously with $3 \mathrm{mg}$ Depo-Provera (long-lasting progestin) in PBS. Six days later, the mice were anesthetized and challenged by intra-vaginal administration with a lethal dose of $9 \times 10^{4}$ plaque forming units (PFU) of HSV-2 strain 333.

\subsection{HSV-2 plaque assay}

Three days after HSV-2 challenge, the mice were anesthetized and vaginal fluids collected. This was done by pipetting $40 \mu \mathrm{l}$ of sterile Hank's balanced salt solution (HBSS) (Gibco) numerous times in and out of the vagina. Two washes from each mouse were pooled in a total volume of $1 \mathrm{ml}$ and frozen at $-80^{\circ} \mathrm{C}$. HSV-2 replication was determined by plaque assay of confluent green monkey kidney cell monolayers in Nunclon Delta 6-well plates (Nunc). The vaginal washes were diluted 1:10 in HBSS and added to the cells, which were left for adsorption for $1 \mathrm{~h}$ at room temperature. Methylcellulose solution (1\%) (Sigma Aldrich) in Iscove's medium (Sigma Aldrich) containing newborn calf serum (2\%) (Sigma Aldrich) and penicillin-streptomycin (0.75\%) (Sigma Aldrich) was then added to each well. The plates were incubated for three days at $37^{\circ} \mathrm{C}$ in $5 \% \mathrm{CO}_{2}$. After three days the cells were stained with a $1 \%$-solution of crystal violet. Plaques were counted using a light-microscope and virus replication data shown as PFU. All samples were analyzed in duplicates.

\subsection{Monitoring of inflammation and disease}

Following HSV-2 challenge, mice were examined daily for vaginal inflammation and neurological illness. The severity of the disease was graded as: $0=$ healthy, $1=$ genital erythema, 2=moderate genital inflammation, $3=$ severe genital lesions and $4=$ hind limb paralysis. Mice were euthanized as soon as they displayed signs of severe illness. The monitoring and scoring procedure was performed for 20 days.

\section{Results}

\subsection{TMV antigen construct}

To express the different antigens, we took advantage of a TMV vector that contained sequences encoding the plant viral coat protein (CP) subgenomic promoter, coat protein 
coding sequence and in addition a 3 '-nontranslated region originating from tobacco mild green mosaic virus, denoted TMGMV (Shivprasad, et al, 1999). This vector was used because it has earlier been shown to mediate expression of an inserted transgene to a level corresponding to as much as $10 \%$ (dry weight) of the total protein in an infected leaf (Shivprasad, et al, 1999). In addition, the virus has a rod-shape morphology, which allows the insertion of the relatively large sizeHSV-2-CP gene fusions. To engineer the TMV-TMGMV constructs (here denoted as the TMV constructs) we purified DNA fragments containing the HSV-2 gD and HSV-2 VP16 sequences and fused them to the strong TMV coat protein promoter. Thus, when introduced into the appropriate host, the fusion proteins translated from the expressed HSV-2 gD and VP16 recombinant viral RNA sequences will be displayed on the surface of the virus-like particles at high density. A schematic diagram representing the resulting recombinant TMV vectors carrying the gD, VP16 and GFP constructs, respectively is presented in Fig.1.

\subsection{Infectivity of recombinant $T M V$}

Using the recombinant TMV vectors as a templates, RNA transcripts were synthesized in vitro, capped with $\mathrm{m} 7 \mathrm{G}(5) \mathrm{ppp}(5) \mathrm{G}$ and the resulting products were used to inoculate the three upper leaves of young, four-leaf stage N.benthamiana plants. Symptoms indicative of TMV infection were first visible as mild yellow spots at 5-7 days post infection (DPI) (data not shown). The infection progressed continuously, became systemically infected and typically developed green mosaic and severe curling of the leaves at stages 14-21 DPI. This phenotype was never observed in non-infected plants (Fig. 2 A-C). Leaf samples were collected from infected plants at the 15 DPI stage for further analyses, a stage at which all three recombinant TMV:HSV-2 constructs has given rise severe symptoms (Fig. 2 D - F).

\subsection{Reverse transcription-PCR (RT-PCR)}

To confirm the expression of also the gD and VP16 antigens on the RNA level, RTPCR assays were performed utilizing forward primers specific for either gD or VP16 sequences and reverse primers specific for the TMV vector. Total RNA from individual $N$. benthamiana plants, each infected with one of the recombinant TMV vectors, was isolated from leaves at stage $15 \mathrm{DPI}$ and used as templates to produce the corresponding DNA. Several independent PCR amplifications were then done utilizing forward primers specific for either gD or VP16 sequences and reverse primers specific for TMV vector sequences. And in every case; amplified fragments of the expected sizes 
(1115 bp for gD and $1590 \mathrm{bp}$ for VP16) were obtained (Fig. 4). This result showed that the recombinant TMV RNA is infectious, replicates in the tobacco host and expresses the engineered $\mathrm{gD}$ and VP16 antigen RNA.

\subsection{Western blot analysis of the recombinant HSV-gD and VP16 antigens}

To demonstrate that the recombinant viruses expressed the introduced construct also at the protein level, leaves from plants infected with the TMV:GFP construct were first tested for GFP mediated light emission by fluorescence microscopy. This showed that leaves from infected plants strongly expressed the GFP gene (Fig. 3). Total protein was extracted from infected leaves from each of the recombinant TMV-vector transfections at $15 \mathrm{DPI}$ and expression of the HSV-2 gD and VP16 proteins was confirmed by western blot analysis utilizing either a general anti-TMV coat protein (CP) polyclonal antibody, or specific mouse monoclonal antibodies raised against the HSV-gD and HSV-VP16 antigens. This showed that the TMVCP, HSV gD and HSV VP16 proteins were present in the plant leaf extracts at DPI 15 (Fig. 5 and Fig. 6). To determine the optimal time point for protein isolation, samples from locally and systemically infected leaves were collected at the 7,15 and 21 DPI stages (data not shown) and tested with the HSV-gD and HSV-VP16 antibodies. Bands of the expected sizes could then be detected on the gels although the intensity between the specific bands varied. The highest expression was at $15 \mathrm{DPI}$. No specific bands could be detected in samples collected at $7 \mathrm{DPI}$ or in samples from healthy plants (Fig.5).

\subsection{ELISA experiments}

Leaf samples were collected at $15 \mathrm{DPI}$ and ELISA tests were performed on crude extracts using the same anti-TMV polyclonal, HSV-gD monoclonal or HSV-VP16 monoclonal antibodies as mentioned above. This confirmed that $N$. benthamiana plants infected with recombinant TMV transcripts expressed the gD and VP16 antigens. Furthermore, to demonstrate that the TMV carries the recombinant $\mathrm{gD}$ and VP16 HSV-2 antigens on the surface, the TMV virus particles were first captured on ELISA plates coated with anti TMV polyclonal antibodies and detected with the anti-gD and anti-VP16 monoclonal antibodies. This showed that plants infected with the recombinant TMV were positive (Table 1) and that the gD and the VP16 were exposed on the surface of the recombinant TMV particle.

\subsection{Immunogenicity of plant-gD protein in mice}


To investigate the immunogenicity of the plant-produced $\mathrm{gD}$ protein in mice, a group of female $\mathrm{C} 57 \mathrm{~B} \mathrm{~B} / 6$ mice were immunized intranasal with either the plant-or $\mathrm{CHO}$ cellproduced gD protein in combination with $\mathrm{CpG} 1826$ as adjuvant. As shown in Fig 7A, sera obtained from mice immunized with plant-gD or $\mathrm{CHO}-\mathrm{gD}$ protein had high titers of $\mathrm{gD}$ specific $\lg G$ antibodies as compared to those of non-immunized control mice.

Next, it was tested in a mouse model if immunization with the plant-gD protein together with CpG1826 adjuvant could induce protection of primary genital herpes infection in a similar way as the $\mathrm{CHO}-\mathrm{gD}$ protein. To address this, mice were immunized with either the plant-gD/adjuvant or the $\mathrm{CHO}-\mathrm{gD} /$ adjuvant mixture. This was followed six days later by a vaginal challenge of a lethal dose of HSV-2 virus strain 333. As a control, non-immunized mice were also infected with the virus. It was found that the control mice had high titers of HSV-2 in their genital secretions three days after the challenge and that the majority of the mice showed so severe symptoms of the disease that they required euthanasia (Fig. 7B-D). The CHO-gD immunized group, on the other hand, showed low or no vaginal HSV-2 replication and all mice survived the challenge. Interestingly, the plant-gD immunized group showed an even higher protection than the CHO-gD group with virtually no vaginal HSV-2 replication. All animals remained symptom free for the duration of the entire experiment i.e., 20 days (Fig. 7B-D). These data indicate that similar to the mammalian-produced $\mathrm{gD}$ protein, the plant-gD protein is highly immunogenic and induce protection against an otherwise lethal vaginal HSV-2 challenge in mice.

\section{Discussion}

Genital herpes is a prevalent sexually-transmitted disease with a major socioeconomic burden in both the developing and the developed world. In spite of several efforts, no vaccine is currently available to counter genital herpes. In addition to the need of developing novel immunization technologies, a production of larger quantities of HSV-2 antigens at a reasonable cost and quality would greatly facilitate the introduction of a HSV vaccine in the developing countries. We suggest that a plantbased HSV-antigen expression system could provide one such alternative.

To produce an antigen in a plant system, the antigen-encoding gene can either be stably introduced into the nuclear or chloroplast DNA (Ahmad et al, 2012), or into an infectious virus that when infecting the plant will mediate a transient expression of the antigen gene. Stably expressed plant-derived vaccines, especially edible vaccines, do 
not require costly fermentation and purification systems, complicated formulations mechanisms or storage at cool or freezing temperatures. Transient systems have the advantage that the expression of the recombinant protein is at much higher levels than what can be obtained from stable systems (Green et al., 2009). All plant expression systems have the advantage that no unknown animal pathogens can present and contaminate the system. Thus, purification of the antigen will be easier and safer, although the degree of purification required depends on nontoxic compounds are present in the plant host.

For transient expression, TMV-based systems have proven to be very useful. TMV is a filamentous virus and can therefore more easily accept exogenous DNA compared to icosahedral viruses where there is a severe space limit on the size of the extra genetic material to be introduced. In addition, although TMV is an RNA virus that replicates in the cytosol, it can be engineered as DNA, which greatly simplifies the necessary cloning steps. The engineered viral DNA is then introduced into plant cells, transported to the nucleus, transcribed to infectious viral RNA molecules that are exported out of the nucleus and translated. Alternatively, the DNA construct can be transcribed and capped already in vitro and the formed infectious RNA molecules directly introduced into the leaf cytoplasm by rubbing. TMV-based systems have successfully been used to express hepatitis B core antigen at levels of up to 1-2 $\mathrm{mg}$ per $\mathrm{g}$ fresh leaf weight in $N$. benthamiana (Musiychuk et al., 2007). In so called "Launch Recombinant Viral Vector Technologies" a range of other genes, including influenza targeting virus antigen genes, have been expressed at very high levels, again in N. benthamiana (Shoji et al., 2009). Other advantages with TMV-based transient expressions systems are that, since TMV is a mechanically transmitted virus, there are no vectors present in nature that can transmit spread the virus into nature. Therefore extra precautions due to possible biohazards are not an issue. In addition, recombinant TMV viruses could only survive in the natural environment for a very short period of time since they rapidly reverse to wild type of TMV (Hammond et al., 1999, Shivprasad et al., 1999). Finally, the virus is not present in pollen and seeds and therefore a vertically spread is not possible either.

In this work we took advantage of a TMV-based vector system, inserted HSV-2 gD and VP16 antigen genes and transiently expressed these in tobacco plants. We collected leaves from infected plants and verified the presence of the antigens in crude extract of these leaves by Western blot and ELISA. Furthermore, as a proof of concept, by intranasally immunizing mice with plant-produced $\mathrm{gD}$ antigen in combination with 
CpG 1826 adjuvant we demonstrated that our plant-gD preparation was immunogenic in mice, similar to $\mathrm{CHO}$-produced gD protein, High titers of gD-specific $\lg \mathrm{G}$ antibody was detected in mice sera and most importantly, the plant gD immunized mice showed a complete protection against a lethal vaginal HSV-2 challenge. This protective effect was even higher than that obtained with $\mathrm{CHO}$-produced $\mathrm{gD}$ protein that was used as a positive control (Persson et al, manuscript in preparation).

Besides its possible value as a vaccine candidate, due to the high antigenicity of the plant- produced gD, it could potentially be used in a simple and inexpensive ELISA based diagnostic tool for HSV-2. Since gD can be expressed on a large scale in plants, it could potentially also be used to capture anti-HSV-2 gD antibodies from the blood serum of infected patients through indirect ELISA.

In summary, we report here the development of a TMV-based transient expression system for production of HSV-2 proteins. Furthermore, we show that the biological activity of the plant-gD preparation is highly immunogenic and protective against an experimental vaginal challenge with HSV-2 in mice and protect these animals from genital herpes.

\section{Acknowledgement}

The authors acknowledge Dr. Shailaja Shivprasad from Farunhofer Centre for Molecular Biotechnology, DE, USA, who kindly provided the 30B hybrid TMV-TMGMV vector and the GFP clone. The authors are also grateful to Karolina Thörn for technical assistance with the mouse experiments. We thank the Swedish Research Council (VR), VR-MENA, Sida-Sarec and the Swedish Institute (SI) for financial support.

\section{References}

Abd-Elshafy, D.N., Mahmoud M. Bahgat, 2017. Herpes Simplex virus type 1 persistence in water from different sources. J. of Virol. Sci., Vol. 2: 114-123.

Aboul-Ata, A.A.E., Vitti, A., Nuzzaci, M., Ahmed K. El-Attar, Piazzolla, G., Tortorella, C., Harandi, A.M., Olson, O., Wright, S.A., Piazzolla, P. 2014. Plant-based vaccines: Novel and low-cost possible route for Mediterranean innovative vaccination strategies. Advances in Virus Research; Volume 89, Pages 1-37.

Ahmad, P., Ashraf, M., Younis, M., Hu, X., Kumar, A., Akram, N.A., Al-Qurainy, F., 2012. Role of transgenic plants in agriculture and biopharming. Biotechnol. Adv., 30(3): 524540.

Ausubel, F.M., Brent, R., Kingston, R.E., Moore, 1999. Short protocols in molecular biology. John Wiley \& Sons 4th edition; NY

Awasthi, S., Balliet, J.W., Flynn, J.A., Lubinski, J.M., Shaw, C.E., Di Stefano, D.J., Cai, M., Brown, M., Smith, J.F., Kowalski, R., Swoyer, R., Galli, J., Copeland, V., Rios, S., Davidson, R.C., Salnikova, M., Kingsley, S., Bryan, J., Casimiro, D.R., Friedman, H.M. 
2014. Protection provided by a herpes simplex virus 2 (HSV-2) glycoprotein C and D subunit antigen vaccine against genital HSV-2 infection in HSV-1-seropositive guinea pigs. J. Virol., 88(4): 2000-2010.

Bahaa M.A. Abd El-Salam; Eman A.E. Abu-Shady; Azaa A. Mira and Magda A.E. Mohamed, 2017. Detection of Epstein-Barr Virus and Herpes Simplex Virus Type-1 in Tonsils or Adenoids. J. of Virol. Sci., Vol. 2: 71-78.

Basse, G., Mengelle, C., Kamar, N., Ribes, D., Selves J., Cointault O., Suc B., Rostaing L., 2008. Disseminated herpes simplex type-2 (hsv-2) infection after solid-organ transplantation. Infection, 36(1): 62-64.

Bernstein, D.L. and Stanberry, L.R. 1999. Herpes simplex virus vaccines. Vaccine, 17(14): 1681-1689

Claire A. Penney, David R. Thomas, Sadia S. Deen, Amanda M. Walmsley. 2011. Plantmade vaccines in support of the Millennium Development Goals. Plant Cell Reports, 30(5):789-798.

Chan, H-T and Henry Daniell, 2015. Plant-made oral vaccines against human infectious diseases-Are we there yet? Plant Biotechnology Journal, 13: 1056-1070

Daniell, H., Singh, N.D., Mason, H., Streatfield, S.J., 2009. Plant made vaccine antigens and biopharmaceuticals. Trends Plant Sci., 14(12): 669-679.

Davison, A.J., Eberle, R., Ehlers, B., Hayward, G. S., McGeoch D. J., Minson A. C., Pellett P. E., Roizman B., Studdert M. J., and Etienne Thiry 2009. The order Herpes virales. Arch. Virol., 154(1): 171-177.

Dutton, J.L., Li, B., Woo, W.P., Marshak, J.O., Xu, Y., Huang, M.L., Dong, L., Frazer, I.H., Koelle, D.M. 2013. A Novel DNA Vaccine Technology Conveying Protection against a Lethal Herpes Simplex Viral Challenge in Mice. PLoS One, 8(10): e76407.

Edward P Rybicki, 2014. Plant-based vaccines against viruses. Virology Journal, 11:205

El-Attar, A.K., A. M. Shamloul, A.A. Shalaby, B.Y. Riad, A. Saad, H.M. Mazyad, J.M. Keith, 2004. Expression of Chimeric HCV Peptide in Transgenic Tobacco plants infected with Recombinant Alfalfa mosaic virus for development of a plant-derived vaccine against HCV. African Journal of Biotechnology, 3(11): 588-594.

El-Turkey, A., Ahmed K. El-Attar, A. E. Aboulata, B. Othman and k. A. Eldougdoug, 2014. Expression of Recombinant gD2 Protein in Transgenic Tomato plants for development of a plant-derived vaccine against Herpes simplex virus-2. Egyptian J. Virol., Vol. 11 (2): $1-13$.

Glynna, J.R., Biraroa, S. and Weissa, H.A., 2009. Herpes simplex virus type 2: a key role in HIV incidence. AIDS., 23(12): 1595-1598.

Green, B.J., Fujiki, M., Mett, V., Kaczmarczyk, J., Shamloul, M., Musiychuk, K., Underkoffler, S., Yusibov, V., Mett, V. 2009. Transient protein expression in three Pisumsativum (green pea) varieties. Biotechnol. J., 4(2): 230-237.

Guan, Z.J., Guo, B., Huo, Y.L., Guan, Z.P., Dai, J.K., Wei, Y.H., 2013. Recent advances and safety issues of transgenic plant-derived vaccines. Appl. Microbiol. Biotechnol., 97 (7): 2817-2840.

Hammond, J., McGarvey, P., Yusibov, V., 1999. Plant biotechnology: new products and applications. Springer, Berlin Heidelberg New York Tokyo.

Jain, A., Saini, V., Kohli, D.V. 2013: Edible transgenic plant vaccines for different diseases. Curr. Pharm. Biotechnol., 14(6): 594-614.

Jazayeri, J., Soleimanjahi, H., Fotouhi, F., Pakravan, N. 2009. Comparison of intramuscular and footpad subcutaneous immunization with DNA vaccine encoding HSV-gD2 in mice. Comparative Immunology, Microbiology and Infectious Diseases, 32(5): 453-461.

Laere, E., Anna Pick Kiong Ling, Ying PeiWong, Rhun Yian Koh, Mohd Azmi Mohd Lila, and Sobri Hussein, 2016. Plant-Based Vaccines: Production and Challenges. Journal of Botany, Article ID 4928637, 11 pages. http://dx.doi.org/10.1155/2016/4928637 
Lal, P., VG Ramachandran, R Goyal, R Sharma. 2007. Edible vaccines: current status and future. Indian Journal of Medical Microbiology, 25(2): 93-102.

Looker, K.J., Garnett, G.P. and Schmid, G.P. 2008. An estimate of the global prevalence and incidence of herpes simplex virus type 2 infection. Bull World Health Organ, 86(10): 805-812.

Musiychuk, K.; Stephenson, N.; Bi, H.; Farrance, C.E.; Orozovic, G.; Brodelius, M.; Brodelius,P.; Horsey, A.; Ugulava, N.; Shamloul, A.M.; Mett, V.; Rabindran, S.; Streatfield, S.J., Yusibov V. 2007. A launch vector for the production of vaccine antigens in plants. Influenza and Other Respiratory Viruses, 1(1): 19-25.

Nemchinov, L.G., TJ Liang, MM Rifaat, HM Mazyad, A Hadidi, JM. Keith. 2000. Development of a plant-derived subunit vaccine candidate against hepatitis $\mathrm{C}$ virus. Arch. Virol., 145(12): 2557-2573.

Regnard, G.L., Edward P. Rybicki, and Inga I. Hitzeroth, 2017. Recombinant expression of beak and feather disease virus capsid protein and assembly of virus-like particles in Nicotiana benthamiana. Virology Journal 14:174

Shivprasad, S., Pogue, G.P., Lewandowski, D.J., Hidalgo, J., Donson, J., Grill, L.K., Dawson, W.O. 1999. Heterologous sequences greatly affect foreign gene expression in tobacco mosaic virus-based vectors. Virology, 255(2): 312-323.

Shoji, Y., Bi, H., Musiychuk, K., Rhee, A., Horsey, A., Roy, G., Green, B., Shamloul, M., Farrance, CE., Taggart, B., Mytle, N., Ugulava, N., Rabindran, S., Mett, V., Chichester, J.A., Yusibov, V., 2009. Plant-derived hemagglutinin protects ferrets against challenge infection with the $\mathrm{A} / \mathrm{Indonesia/05/05}$ strain of avian influenza. Vaccine, 27(7): 1087-1092.

Takeyama, N., Hiroshi Kiyono and Yoshikazu Yuki, 2015. Plant-based vaccines for animals and humans: recent advances in technology and clinical trials. Ther Adv Vaccines, Vol. 3(5-6) 139-154. DOI: 10.1177/2051013615613272

VanBenthem, B.H, Spaargaren, J., van den Hoek, J.A., Merks, J., Coutinho, R.A., Prins, M. 2001. Prevalence and risk factors of HSV-1 and HSV-2 antibodies in European HIV infected women. Sexually Transmitted Infections, 77(2): 120-124.

Yang, M., Haiyan Sun, Huafang Lai, Jonathan Hurtado and Qiang Chen, 2017.Plantproduced Zika virus envelope protein elicits neutralizing immune responses that correlate with protective immunity against Zika virus in mice. Plant Biotechnology Journal, pp. 1-9

Yusibov, V., Streatfield, S. J., Kushnir, N., Roy, G., Padmanaban, A. 2013. Hybrid viral vectors for vaccine and antibody production in plants. Curr. Pharm. Des., 19(31): 55745586. 


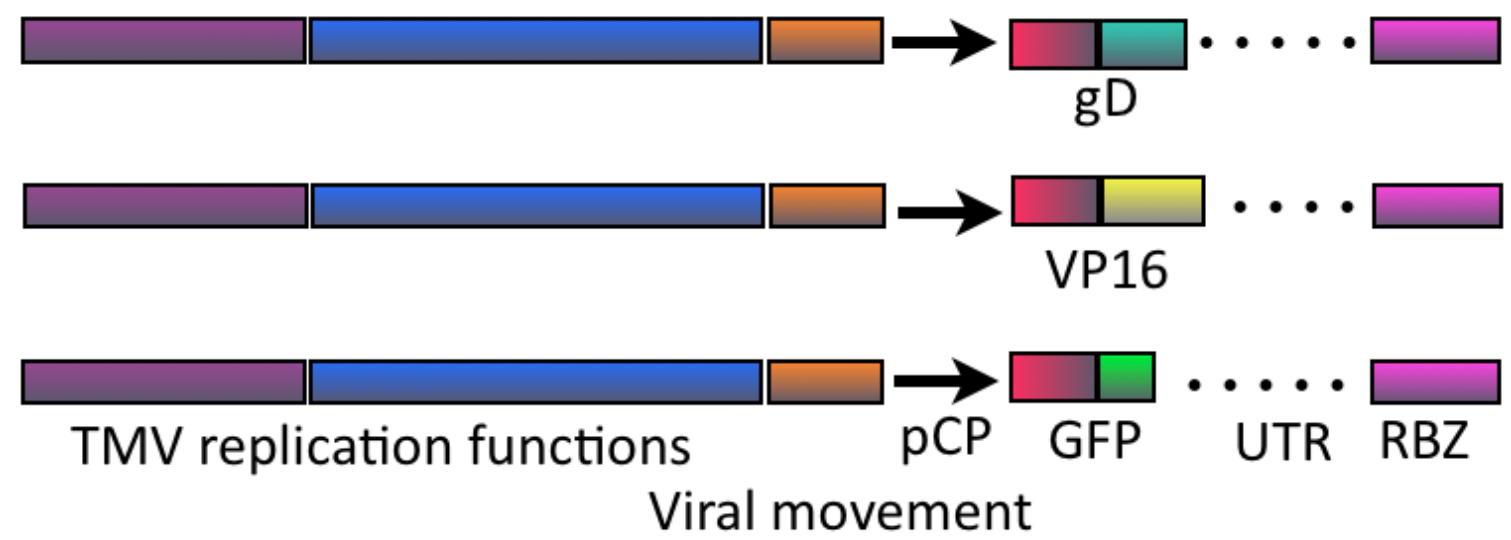

\section{Fig 1: Schematic diagram of the hybrid TMV-based vectors}

Purple and blue bars at the left represent the genes encoding the $126 \mathrm{kDa}$ and $183 \mathrm{kDa}$ proteins required for TMV replication. The brown bar to the right represents the gene encoding the $30 \mathrm{kDA}$ movement protein. $\mathrm{pCP}$ indicates the TMV subgenomic coat protein promoter. gD, VP16 and GFP, respectively indicate the different heterologous sequences that were inserted into the TMV vector. UTR is the untranslated region of TMGMV and RBZ the TTMGMV rybozyme gene.

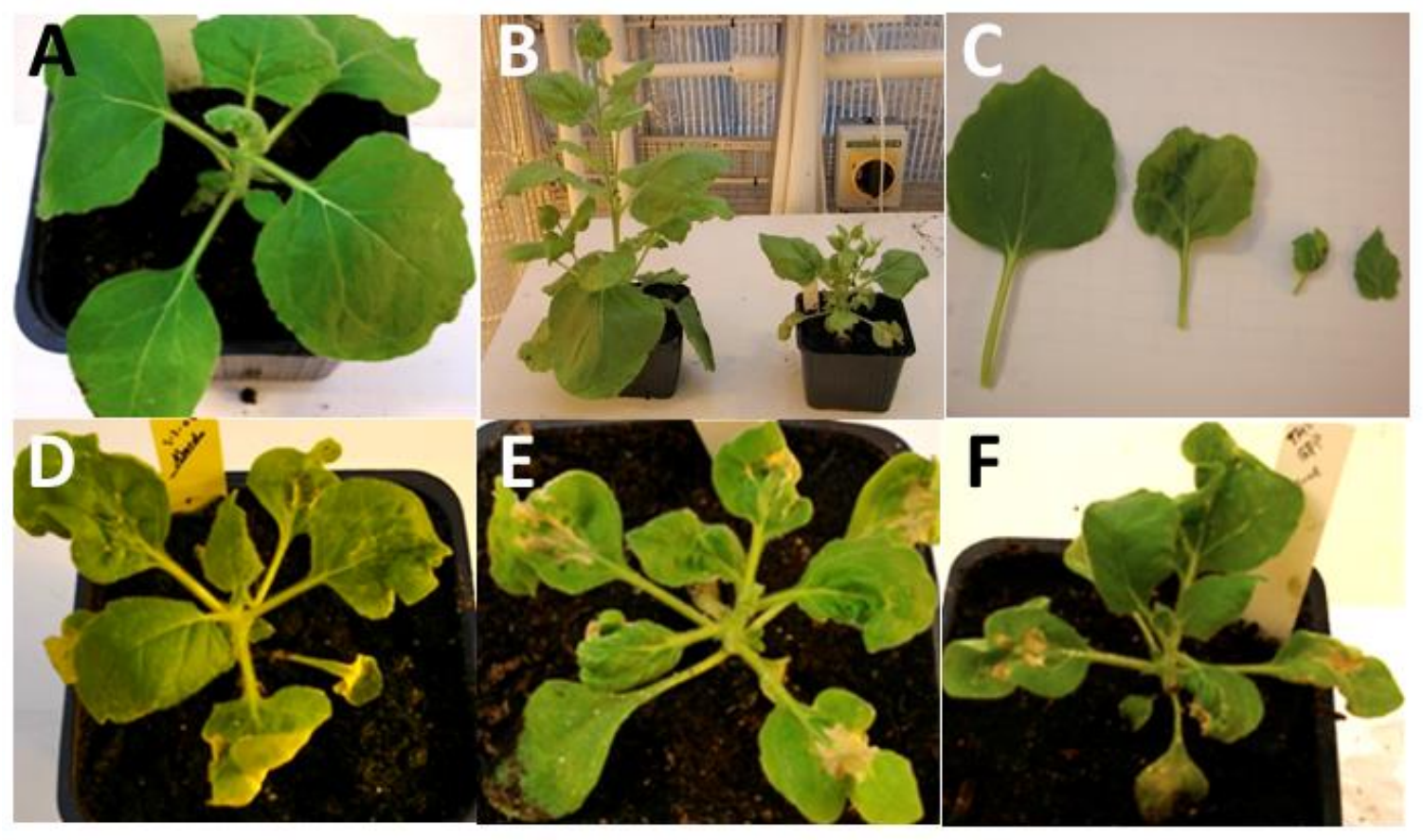

Fig. 2: Chimeric TMV infection of Nicotiana benthamiana plants.

A, Healthy, non-infected plant; B-C, typical symptoms on infected plants; D, gD; E, VP16 and F, GFP expressing plants 


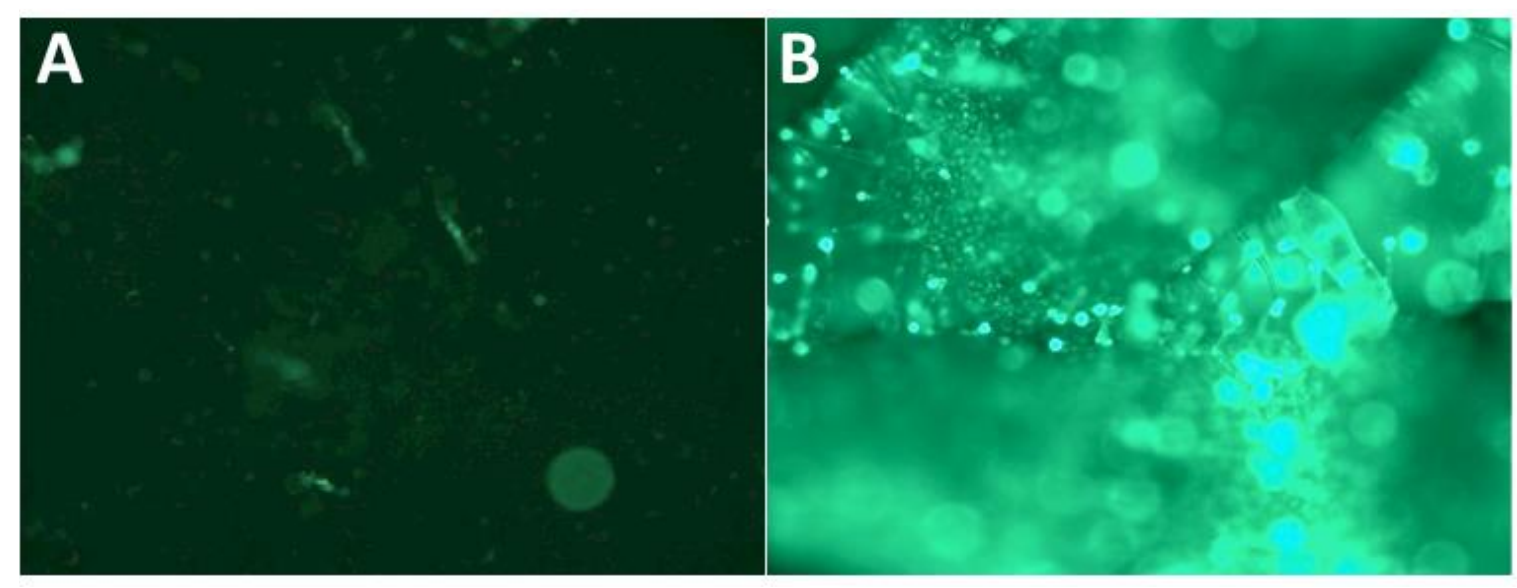

Fig. 3 GFP assay on $\boldsymbol{N}$. benthamiana leaves

A. Non-infected leaves; B, leaves infected with the TMVGFP construct

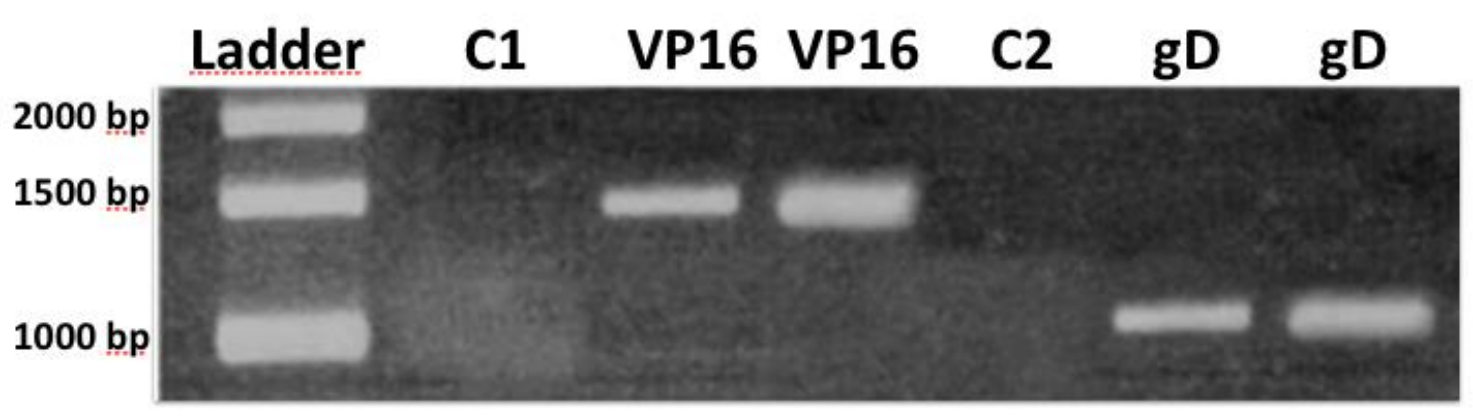

Fig. 4: RT-PCR on total RNA from infected $\boldsymbol{N}$. benthamiana plants

Numbers on left indicates sizes (bp) of the fragments in the DNA ladder. C1; amplification using VP16 specific primers and DNA from non-infected leaves as template; VP16, amplification using VP16 specific primers and DNA from VP16-infected leaves as template; $\mathrm{C} 2$, amplification using $\mathrm{gD}$ specific primers and DNA from non-infected leaves as template; gD, amplification using gD specific primers and DNA from gD-infected leaves as template. VP16 fragment sizes are $1590 \mathrm{bp}$ and gD fragment sizes are $1115 \mathrm{bp}$.
M
C
gD
VP16
GFP

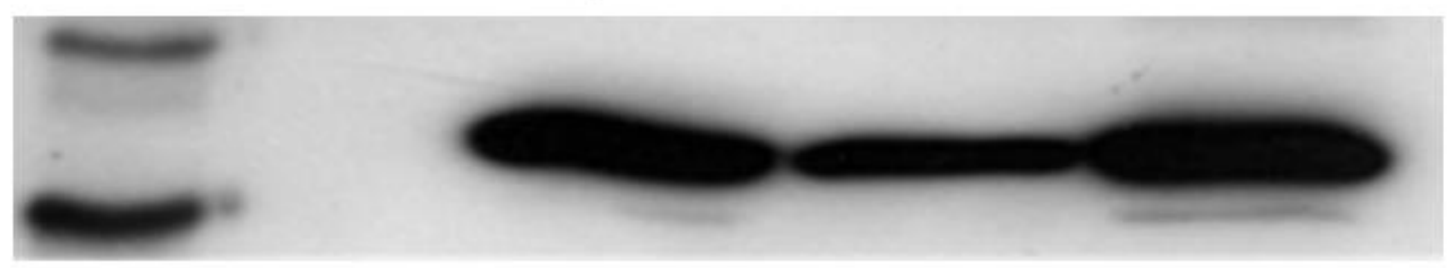

Fig. 5. Western blot analysis of proteins from infected $\boldsymbol{N}$. benthamiana plants

After separating total leaf proteins in by polyacrylamide gel electrophoreses and blotting them to a filter, the filter was incubated with anti-TMV coat protein (CP) polyclonal antibodies. Specific signals obtained from antibodies bound to the filter were developed as 
described in M\&M. M, MagicMark protein standard (Invitrogen); C, plant extract from uninfected control; gD, extract from plants infected with recombinant TMV/gD virus; VP16, extract from plants infected with the recombinant TMV/VP16 virus; GFP, extract from plants infected with recombinant TMV/GFP virus.

A

1

2

C

4

5

6

C

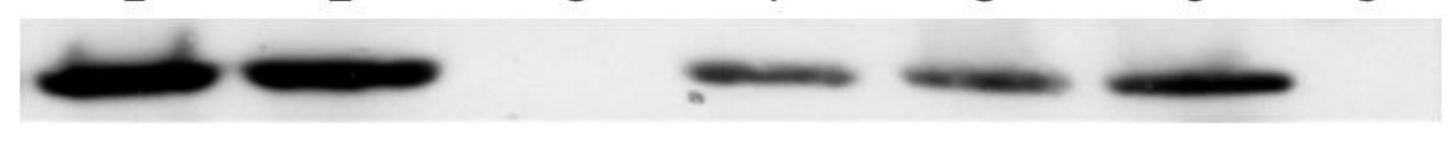

B
C
1
2
$3 \mathrm{C}$
5
6
$7 \quad 8$

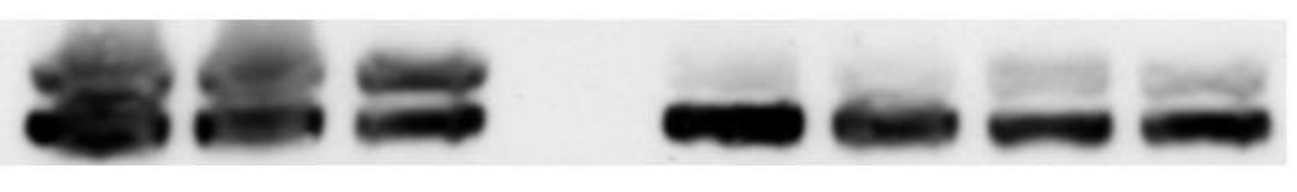

Fig. 6. Western blot analysis of semi-purified proteins from infected $N$. benthamiana plants

A. The membrane was probed with anti-gD monoclonal antibodies. 1, 2 and 4-6, extracts from TMV::gD infected plants; C, extract from non-infected plants.

B. The membrane was probed with anti-VP16 monoclonal antibodies. C, extracts from non-infected plants. 1-3 and 5-8, extracts from TMV::VP16 infected plants

A

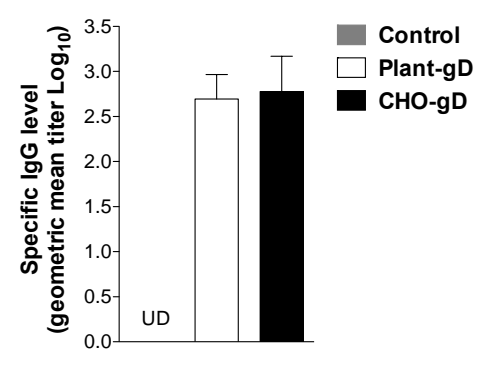

C

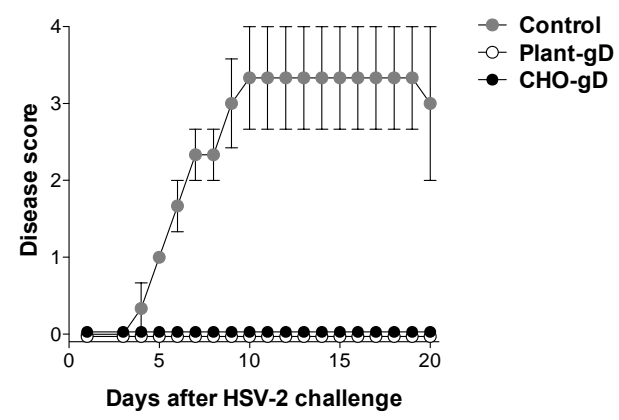

B

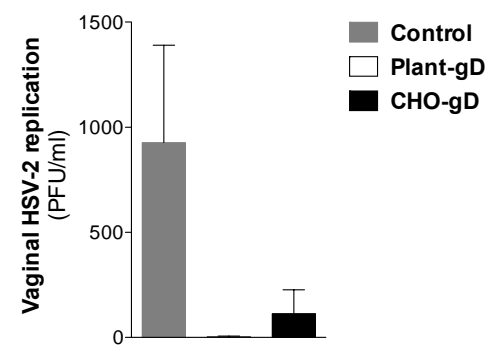

D

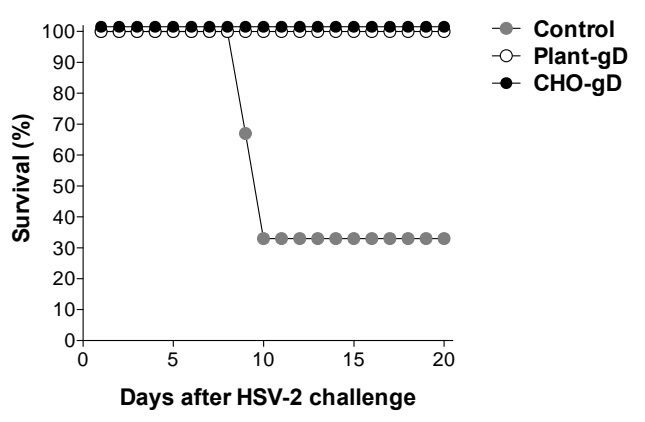


Fig. 7. Immunogenecity of HSV-2gD in both extraction of tobacco vaccine and in fluids of Chinese Hamster Ovary cells.

A. Titter determination of gD-specific IgG in the intranasal immunized mice with either plant- or CHO-induced gD protein and CpG 1826 adjuvant and non-immunized mice as control,

B. PFU/ml assay for HSV-2 replication in immunized mice, that was mentioned above, and followed by a vaginal challenge of HSV-2 virus strain 333 and non-immunized mice were also infected with the virus as control.

C. HSV-2 infection score in both CHO-gD immunized group and plant-gD immunized group and non-immunized mice as control.

D. Survival mice \% in both $\mathrm{CHO}-\mathrm{gD}$ immunized and plant-gD immunized groups against vaginal-HSV-2 replication challenge as control.

Table 1. ELISA analysis of infected plants. Total protein isolated from recombinant TMV/HSV-2gD, TMV/HSV-2VP16 and TMV/GFP as well as from healthy control plant were immobilised in microtiter plates and incubated with monoclonal antibodies MAb:HSV2gD, MAb:HSV-2VP16 and PAb of engineered TMV.

\begin{tabular}{|l|l|l|l|}
\hline Antibody type & MAb of HSV2gD & MAb of HSV-2VP16 & PAb of TMV \\
\hline THV-HSV-2 gD & 0.90 & 0.00 & 0.79 \\
\hline TMV-HSV-2 VP16 & 0.00 & 0.65 & 0.70 \\
\hline TMV-GFP & 0.00 & 0.00 & 1.00 \\
\hline Healthy plant control & 0.05 & 0.05 & 0.05 \\
\hline
\end{tabular}

*Anti-TMV polyclonal antibody was used as a primary capturing agent; anti-gD and/or anti VP16 mouse monoclonal antibodies (MAbs) and/or anti-TMV polyclonal antibody (PAb) was used as a secondary antibody. Cutoff OD value of 0.0106 was the background signal from the buffer controls. Healthy $N$. Benthamiana plants (HPC) were used as a negative control. 